\title{
Performance Analysis of Voice/Data Cellular CDMA With SIR-Based Admission Control
}

\author{
S. Anand, Student Member, IEEE and A. Chockalingam, Senior Member, IEEE
}

\begin{abstract}
In this paper, we analyze the performance of a signal-to-interference ratio (SIR)-based admission control strategy on the uplink in cellular code-division multiple-access (CDMA) systems with voice and data traffic. Most studies in the current literature to estimate CDMA system capacity with both voice and data traffic do not take into account admission control based on SIR constraints. Here, we present an analytical approach to evaluate the outage probability for voice traffic, the average system throughput, and the mean delay for data traffic in a voice/data CDMA system, which employs an SIR-based admission control. We make two main approximations in the voice call outage analysis-one based on the central limit theorem (CLT) and the other based on the Fenton's method. We apply the Fenton's method approximation to compute the retransmission probability and the mean delay for data traffic, and the average system throughput. We show that for a voice-only system, a capacity improvement of about $30 \%$ is achieved with the SIR-based admission control as compared with the code availability-based admission control. For a mixed voice/data system with 10 Erlangs of voice traffic, an improvement of about $40 \%$ in the mean delay for data is shown to be achieved. Also, for a mean delay of $50 \mathrm{~ms}$ with 10 Erlangs of voice traffic, the data Erlang capacity improves by about $50 \%$.
\end{abstract}

Index Terms-Admission control, cellular code-division multiple-access (CDMA), voice/data traffic.

\section{INTRODUCTION}

C ODE-DIVISION multiple-access (CDMA) cellular systems have been known to perform better than the channelized systems in terms of system capacity or carried traffic [1]. Several studies analyzing the outage performance and capacity of CDMA systems have been reported in the literature [2]-[8]. While [2]-[4] consider voice-only CDMA systems, [5]-[8] consider CDMA systems with mixed voice/data traffic. In [5], Mandayam et al. considered a multiple-cell system and computed the voice call outage probability in the presence of data traffic sources, but without admission control. Admission control in cellular CDMA systems based on a threshold on the number of users allowed in the system was studied in [2] and [3]. Evans and Everitt in [2], evaluated the outage probability in voice-only cellular CDMA systems by making two main approximations based on the central limit theorem (CLT) and the Chernoff bound (CB). They showed that the CLT

Manuscript received October 5, 2002; revised May 13, 2003. This work was supported in part by the Indo-French Centre for Promotion of Advanced Research, New Delhi, under Project 2900-IT. This work was presented in part at the IEEE WCNC 2002, Orlando, FL, March 2002, and in part at the IEEE GLOBECOM 2002, Taipei, Taiwan, November 2002.

The authors are with the Department of Electrical Communication Engineering, Indian Institute of Science, Bangalore 560 012, India (e-mail: anand@ hilbert.ece.iisc.ernet.in; achockal@ece.iisc.ernet.in).

Digital Object Identifier 10.1109/JSAC.2003.814756 approximation gave a lower bound on the outage probability and the CB approximation gave an upper bound. Karmani and Sivarajan in [4], applied Edgeworth expansion to obtain better approximations to the outage probability as compared with the bounds given in [2]. Admission control based on the availability of spreading codes has been extensively studied [6]-[8]. However, the above studies on admission control in CDMA systems do not consider signal-to-interference ratio (SIR) constraints before admitting a call.

In this paper, we analyze the performance of an SIR-based admission control policy which allows only those incoming calls that not only have a spreading code available for transmission, but also whose interference-to-signal $(I / S)$ levels at their respective base stations are below a specified threshold. Such a newly admitted call could cause the $I / S$ at any of the other cells to go above the threshold. We define this event as an outage and define the outage probability as the probability that a newly admitted call causes outage to any of the ongoing calls in the system. For data traffic, this outage results in retransmission of the data, due to which we call the outage probability for data traffic as the retransmission probability. By allowing only those calls that see an acceptable $I / S$ level at their respective base stations, the outage probability for voice traffic and the retransmission probability for data traffic can get reduced. The reduction in the retransmission probability for data traffic, in turn, results in reduced mean delay and increased throughput and, hence, in an increased capacity for a specified mean delay or voice call outage probability.

We first consider a voice-only cellular CDMA system and derive analytical expressions for the outage probability, taking into account the SIR-based admission control [9]. We model the system as an $M / G / \infty$ queue and make two main approximations in the outage analysis - one based on the CLT and the other based on the Fenton's method. We then consider a mixed voice/data CDMA system which employs SIR-based admission control, and develop an analytical approach to evaluate the performance of the mixed voice/data system [10]. We derive expressions for: 1) the outage probability of voice calls; 2 ) the average system throughput; and 3) the mean delay performance for data traffic. For deriving the voice call outage probability, we use the Fenton's method approximation. For deriving the mean delay for data traffic, we model the system as a single virtual buffer, where all the buffered data (at all the users in all the cells) are queued in the order of their arrival epochs. We compute the mean delay for the first data burst that departs from this virtual buffer. We then model the rest of the buffer as an $M / G / 1$ queue with a mean service time equal to the mean delay of the first departing data burst. We show that significant improvement in the 
system capacity is possible with SIR-based admission control as compared with code availability (CA)-based admission control.

The rest of the paper is organized as follows. In Section II, we present the system model. Section III provides the analysis to derive expressions for the voice call outage probability, the average system throughput, and the mean delay for data traffic. Section IV presents the results and discussions. Section V provides the conclusions.

\section{SYSTEM MODEL}

Consider a voice/data CDMA cellular system with $N=61$ circular cells. The objective is to develop an analytical approach to evaluate the performance of this system with an SIR-based admission control on the uplink. The performance measures of interest are the voice-call outage probability, the average system throughput, and the mean delay for data traffic.

Voice calls are assumed to be of circuit-switched type. Each voice call uses a spreading code for transmission. The assigned code is held for the entire duration of the call, after which it is released (i.e., for voice, codes are allocated on a call-by-call basis). Data traffic, on the other hand, is assumed to arrive in bursts. Spreading codes are allocated and released on a burst-byburst basis.

A voice call or a data burst originating from a user is admitted into the system if: 1) spreading codes are available for allocation and 2) the $I / S$ ratio measured at the corresponding base station is less than a desired threshold. The $I / S$ thresholds for voice and data are $\epsilon_{v}$ and $\epsilon_{d}$, respectively, which can be chosen based on the transmission rates of the voice and data traffic. Voice calls which are not admitted are blocked, and data bursts which are not admitted are buffered and are admitted when both conditions 1), as well as 2) mentioned above are satisfied following the departure of an ongoing call in the system.

For the buffered data (at all the users in all the cells), the system behaves like a single virtual queue as follows. All the base stations in the system coordinate among themselves and keep track of a virtual queue of data bursts, by assigning an index to each buffered data burst. The index is assigned based on the increasing order of the arrival epochs of the buffered data bursts. When a code becomes free and the $I / S$ conditions become favorable following the departure of an ongoing call, the base stations allow only the user, which has the data burst with the least index among the data bursts which have favorable $I / S$ condition, to transmit using its assigned code. We make the following assumptions to carry out the performance analysis.

- Each cell has radius $R$ and the base station is located at the center of the cell.

- Each cell has a maximum of $n=64$ spreading codes available for allocation.

- Users are uniformly distributed over the area of each cell and are assumed to have no mobility.

- At any given time, each active user is assumed to generate either voice or data traffic, but not both simultaneously.

- The voice call arrival process in each cell is Poisson with mean arrival rate $\lambda_{v}$. The voice call holding times are expo- nentially distributed with mean $\mu_{v}^{-1} \mathrm{~s}$. We define the mean voice traffic load $\rho_{v}$ as $\rho_{v} \triangleq \lambda_{v} / \mu_{v}$ Erlangs/cell.

- The data burst arrival process in each cell is Poisson with mean arrival rate $\lambda_{d}$. The data burst lengths are exponentially distributed with mean $\mu_{d}^{-1} \mathrm{~s}$. We define the mean data traffic load $\rho_{d}$ as $\rho_{d} \triangleq \lambda_{d} / \mu_{d}$ Erlangs/cell.

- The $I / S$ thresholds for admitting voice calls and data bursts are $\epsilon_{v}$ and $\epsilon_{d}$, respectively.

- Voice calls are transmitted at a rate $r^{(v)} \mathrm{b} / \mathrm{s}$ and data bursts are transmitted at a rate $r^{(d)} \mathrm{b} / \mathrm{s}$. We consider $r^{(d)}=k_{d} r^{(v)}$, $k_{d}>1$, so that $\epsilon_{v}=k_{d} \epsilon_{d}$.

- The signal undergoes distance attenuation, shadow loss and multipath Rayleigh fading. The path loss exponent is taken to be four. The shadow loss is assumed to be log-normally distributed of the form $10^{-\psi / 10}$, where $\psi \sim \mathcal{N}\left(0, \sigma^{2}\right)$. For voice traffic, the multipath Rayleigh fading is assumed to be averaged out due to the long holding times of voice calls.

- We assume perfect power control for voice calls and no power control for data bursts. That is, each base station receives unit power from all the voice users attached to it irrespective of their positions in the cell.

\section{PERformance AnAlysis}

In cellular CDMA, because of universal frequency reuse, the interference in a given cell is due to the in-cell and the other-cell active users. Here, we assume that the interference seen by a base station is due to the users in its first tier of neighboring cells, i.e., we ignore the interference due to the users located in the cells other than the first tier neighboring cells as negligible. ${ }^{1}$ The number of interferers with voice traffic seen by cell $k, \Delta_{k}^{(v)}$, can be written as

$$
\Delta_{k}^{(v)}=\Delta_{I_{k}}^{(v)}+\Delta_{O_{k}}^{(v)}
$$

where $\Delta_{I_{k}}^{(v)}$ is the number of in-cell voice interferers and $\Delta_{O_{k}}^{(v)}$ is the number of neighboring-cell voice interferers to cell $k$. Similarly, the number of interferers with data traffic seen by cell $k, \Delta_{k}^{(d)}$, is given by

$$
\Delta_{k}^{(d)}=\Delta_{I_{k}}^{(d)}+\Delta_{O_{k}}^{(d)}
$$

where $\Delta_{I_{k}}^{(d)}$ is the number of in-cell data interferers and $\Delta_{O_{k}}^{(d)}$ is the number of neighboring-cell data interferers to cell $k$.

Let $I_{k}^{\prime}\left(\Delta_{k}^{(v)}, \Delta_{k}^{(d)}\right)$ denote the $I / S$ at the base station of cell $k$ due to $\Delta_{k}^{(v)}$ voice interferers and $\Delta_{k}^{(d)}$ data interferers. $I_{k}^{\prime}\left(\Delta_{k}^{(v)}, \Delta_{k}^{(d)}\right)$ can then be written as

$$
I_{k}^{\prime}\left(\Delta_{k}^{(v)}, \Delta_{k}^{(d)}\right)=\Delta_{I_{k}}^{(v)}+I_{k}\left(\Delta_{O_{k}}^{(v)}, \Delta_{k}^{(d)}\right)
$$

where the first term is due to the perfectly power controlled in-cell voice interferers and the second term is due to the neighboring-cell voice interferers and all the data interferers.

\footnotetext{
${ }^{1}$ Henceforth, we use the term neighboring cells to mean the first tier of cells around the cell-of-interest.
} 
$I_{k}\left(\Delta_{O}^{(v)}, \Delta_{k}^{(d)}\right)$ can be written, in terms of distance attenuation, shadow loss and multipath Rayleigh fading loss, as

$$
\begin{aligned}
I_{k}\left(\Delta_{O_{k}}^{(v)}, \Delta_{k}^{(d)}\right)= & \frac{1}{k_{d}} \sum_{\substack{i \in S_{k} \\
i \neq k}} \sum_{j=1}^{\Delta_{i k}^{(v)}} \frac{D^{4}\left(U_{j i}^{v}, B_{i}\right) 10^{-\frac{\psi_{j i}^{v}}{10}}}{D^{4}\left(U_{j i}^{v}, B_{k}\right) 10^{-\frac{\psi_{j k}^{v}}{10}}} \\
& +\sum_{i \in S_{k}} \sum_{j=1}^{\Delta_{i k}^{(d)}} D^{-4}\left(U_{j i}^{d}, B_{k}\right) 10^{-\frac{\psi_{j k}^{d}}{10}} \alpha_{j k}^{2}
\end{aligned}
$$

where $S_{k}$ denotes the set of cells containing cell $k$ and its neighboring cells, and $\Delta_{i k}^{(v)}$ and $\Delta_{i k}^{(d)}$ are the number of voice and data interferers, respectively, in cell $i$ to cell $k$. Note that $\Delta_{O_{k}}^{(v)}=\sum_{\substack{i \in S_{k} \\ i \neq k}} \Delta_{i k}^{(v)}$ and $\Delta_{k}^{(d)}=\sum_{i \in S_{k}} \Delta_{i k}^{(d)}$. Also, $\psi_{j k}^{v}$, $\psi_{j k}^{d} \sim \mathcal{N}\left(0, \sigma^{2}\right)$ correspond to the shadow losses from $j$ th user in cell $i$ to the $k$ th base station for voice and data interferers, respectively. $\alpha_{j k}^{2}$ corresponds to the Rayleigh-fading loss from the $j$ th user in cell $i$ to the $k$ th base station. $D\left(U_{j i}^{v}, B_{k}\right)$ is the distance between the $j$ th voice interferer in cell $i$ and the $k$ th base station, and $D\left(U_{j i}^{d}, B_{k}\right)$ is the distance between the $j$ th data interferer in cell $i$ and the $k$ th base station. The $k_{d}^{-1}$ factor in the first term of (4) accounts for the lesser transmit power for voice users relative to that of the data users, because of the difference in the transmission rates of the voice and data traffic. Note that $I_{k}\left(\Delta_{O_{k}}^{(v)}, \Delta_{k}^{(d)}\right)$ is conditioned on $\Delta_{O_{k}}^{(v)}, \Delta_{k}^{(d)}$, $\alpha_{j k}$ and the location of the interferers and, hence, it needs to be averaged over these variables.

\section{A. Voice-Only System}

In this subsection, we consider a voice-only cellular CDMA system with SIR-based admission control and derive analytical expressions for the voice call outage probability. We make two main approximations in the outage analysis. The approximations are based on: 1) CLT and 2) Fenton's method. The $I / S$ at the base station of cell $k$ in a voice-only cellular CDMA system is obtained by substituting $\Delta_{k}^{(d)}=0$ and $k_{d}=1$ in (3) and (4). Hence, $I_{k}^{\prime}\left(\Delta_{k}^{(v)}\right)$ for a voice-only system can be written as

$$
I_{k}^{\prime}\left(\Delta_{k}^{(v)}\right)=\Delta_{I_{k}}^{(v)}+I_{k}\left(\Delta_{O_{k}}^{(v)}\right)
$$

where

$$
I_{k}\left(\Delta_{O_{k}}^{(v)}\right)=\sum_{\substack{i \in S_{k} \\ i \neq k}} \sum_{j=1}^{\Delta_{i k}^{(v)}} \frac{D^{4}\left(U_{j i}^{v}, B_{i}\right) 10^{-\frac{\psi_{j i}^{v}}{10}}}{D^{4}\left(U_{j i}^{v}, B_{k}\right) 10^{-\frac{\psi_{j k}^{v}}{10}}}
$$

and $\Delta_{O_{k}}^{(v)}=\sum_{\substack{i \in S_{k} \\ i \neq k}} \Delta_{i k}^{(v)}$.

1) Voice-Only System Without SIR-Based Admission Control: In the absence of SIR-based admission control, the outage probability is given by

$$
p_{o}^{*}\left(\epsilon_{v}, \Delta_{O_{k}}^{(v)}\right)=\operatorname{Pr}\left\{I_{k}\left(\Delta_{O_{k}}^{(v)}\right)>\epsilon_{v}\right\} .
$$

We first compute the outage probability conditioned on $\Delta_{I_{k}}^{(v)}$ and then average over $\Delta_{I_{k}}^{(v)}$. We define

$$
\epsilon_{v}^{\prime} \triangleq \epsilon_{v}-\Delta_{I_{k}}^{(v)}
$$

Hence, the outage probability conditioned on $\Delta_{I_{k}}^{(v)}$ when there is no SIR-based admission control, $p_{o}^{*}\left(\epsilon_{v}^{\prime}\right)$, is given by

$$
p_{o}^{*}\left(\epsilon_{v}^{\prime}\right)=\sum_{K} \operatorname{Pr}\left\{I_{k}(K)>\epsilon_{v}^{\prime}\right\} \operatorname{Pr}\left\{\Delta_{O_{k}}^{(v)}=K\right\}
$$

To average (9) over $\Delta_{O_{k}}^{(v)}$, we model the set of all other-cell interferers to a given cell $k$ as a queue length process of an $M / G / \infty$ queue. Hence, $\Delta_{O_{k}}^{(v)}$ is a Poisson random variable with mean $\hat{\rho}_{v}=N_{k} \rho_{v}$, where $N_{k}$ is the number of neighboring cells to cell $k$ (i.e., $N_{k}=6$ ). The probability mass function of $\Delta_{O_{k}}^{(v)}$ is given by

$$
\operatorname{Pr}\left\{\Delta_{O_{k}}^{(v)}=m\right\}=\frac{e^{-\hat{\rho}_{v}} \hat{\rho}_{v}^{m}}{m !}
$$

The outage probability averaged over $\Delta_{I_{k}}^{(v)}$, is then given by

$$
p_{o}^{*}=\sum_{m_{k}=0}^{n} p_{o}^{*}\left(\epsilon_{v}^{\prime}\right) \operatorname{Pr}\left\{\Delta_{I_{k}}^{(v)}=m_{k}\right\} .
$$

A cell with a maximum of $n$ spreading codes can be modeled as an $M / G / n / n$ loss system with $\Delta_{I_{k}}^{(v)}$ as the queue length process. However, for large $n$, this can be approximated by an $M / G / \infty$ system. Hence, the probability mass function of $\Delta_{I_{k}}^{(v)}$ is given by

$$
\operatorname{Pr}\left\{\Delta_{I_{k}}^{(v)}=m\right\}=\frac{e^{-\rho_{v}} \rho_{v}^{m}}{m !}
$$

2) Voice-Only System With SIR-Based Admission Control: With SIR-based admission control, we are interested in the probability that a newly admitted call in cell $i$ causes an outage in cell $k$. The outage probability conditioned on $\Delta_{O_{i}}^{(v)}$, $\Delta_{O_{k}}^{(v)}$ and $\Delta_{I_{k}}^{(v)}$, denoted as $p_{o}\left(\Delta_{O_{i}}^{(v)}, \Delta_{O_{k}}^{(v)}, \epsilon_{v}^{\prime}\right)$, can be written as

$$
\begin{aligned}
& p_{o}\left(\Delta_{O_{i}}^{(v)}, \Delta_{O_{k}}^{(v)}, \epsilon_{v}^{\prime}\right)=\operatorname{Pr}\left\{I_{k}\left(\Delta_{O_{k}}^{(v)}\right)>\epsilon_{v}^{\prime} \mid I_{i}\left(\Delta_{O_{i}}^{(v)}\right)\right. \\
&\left.\leq \epsilon_{v}^{\prime}, I_{k}\left(\Delta_{O_{k}}^{(v)}-1\right) \leq \epsilon_{v}^{\prime}\right\}
\end{aligned}
$$

The above equation gives the probability that a newly admitted call in cell $i$ (because of $I_{i}\left(\Delta_{O_{i}}^{(v)}\right) \leq \epsilon_{v}^{\prime}$ ) causes an outage in cell $k$ (i.e., $I_{k}\left(\Delta_{O_{k}}^{(v)}\right)>\epsilon_{v}^{\prime}$ ) given that there was no outage in cell $k$ before admitting the new call in cell $i$ (i.e., $I_{k}\left(\Delta_{O_{k}}^{(v)}-\right.$ $\left.1) \leq \epsilon_{v}^{\prime}\right)$. We assume that $I_{i}\left(\Delta_{O_{i}}^{(v)}\right)$ is statistically independent of $I_{k}\left(\Delta_{O_{k}}^{(v)}\right)$ to simplify the analysis. Hence, $p_{o}\left(\Delta_{O_{i}}^{(v)}, \Delta_{O_{k}}^{(v)}, \epsilon_{v}^{\prime}\right)$ becomes independent of the cell $i$ in which the call arrives and, hence, can be denoted by $p_{o}\left(\Delta_{O_{k}}^{(v)}, \epsilon_{v}^{\prime}\right)$, which can be written as

$p_{o}\left(\Delta_{O_{k}}^{(v)}, \epsilon_{v}^{\prime}\right)=\operatorname{Pr}\left\{I_{k}\left(\Delta_{O_{k}}^{(v)}\right)>\epsilon_{v}^{\prime} \mid I_{k}\left(\Delta_{O_{k}}^{(v)}-1\right) \leq \epsilon_{v}^{\prime}\right\}$.

Averaging over $\Delta_{O_{k}}^{(v)}$, the outage probability conditioned on $\Delta_{I_{k}}^{(v)}, p_{o}\left(\epsilon_{v}^{\prime}\right)$, is given by

$$
p_{o}\left(\epsilon_{v}^{\prime}\right)=1-\tilde{p}_{o}\left(\epsilon_{v}^{\prime}\right)
$$


where

$$
\tilde{p}_{o}\left(\epsilon_{v}^{\prime}\right)=\prod_{\substack{i \in S_{k} \\ i \neq k}}\left[1-\sum_{m_{i}} p_{o}\left(m_{i}, \epsilon_{v}^{\prime}\right) \operatorname{Pr}\left\{\Delta_{O_{i}}^{(v)}=m_{i}\right\}\right] .
$$

Finally, $p_{o}\left(\epsilon_{v}^{\prime}\right)$ is averaged over $\Delta_{I_{k}}^{(v)}$ to obtain

$$
p_{o}=\sum_{m_{k}=0}^{\infty} p_{o}\left(\epsilon_{v}^{\prime}\right) \operatorname{Pr}\left\{\Delta_{I_{k}}^{(v)}=m_{k}\right\}
$$

In the above, we use the expression in (12) to evaluate $\operatorname{Pr}\left\{\Delta_{I_{k}}^{(v)}=m_{k}\right\}$. It is noted that (12) is valid only in the absence of admission control. However, in order to simplify the analysis, we use this as an approximation for the loads under consideration.

It is noted that the key step in the outage probability computation in the above is the evaluation of (14). In order to evaluate (14), we need to compute the joint probability

$$
P_{j}=\operatorname{Pr}\left\{I_{k}\left(\Delta_{O_{k}}^{(v)}\right)>\epsilon_{v}^{\prime}, I_{k}\left(\Delta_{O_{k}}^{(v)}-1\right) \leq \epsilon_{v}^{\prime}\right\}
$$

and the marginal probability

$$
P_{m}=\operatorname{Pr}\left\{I_{k}\left(\Delta_{O_{k}}^{(v)}-1\right) \leq \epsilon_{v}^{\prime}\right\} .
$$

The marginal probability in (19) can be computed from the outage probability in a voice-only system without SIR-based admission control, given in (7). It is noted that, in [2], Evans and Everitt used approximations based on CLT and CB to evaluate (7). Karmani and Sivarajan in [4], improved the approximations in [2] by applying Edgeworth expansion. The expression in (19) can be computed using any of the above approximations presented in [2] and [4]. To evaluate the joint probability in (18), one can model the random process $I_{k}\left(\Delta_{O_{k}}^{(v)}\right)$ as a continuous state space Markov chain, which can be solved by the theory of random walks. However, this approach is complex. Hence, we adopt simpler methods using CLT approximation and Fenton's method approximation to compute the joint probability $P_{j}$ in (18), which are illustrated in the following sections.

Approximation Using CLT: In order to evaluate the joint probability in (18), the cdfs of $I_{k}\left(\Delta_{O k}^{(v)}-1\right)$ and $I_{k}\left(\Delta_{O k}^{(v)}\right)$ need to be computed. We apply CLT to approximate the other-cell interference $I_{k}\left(\Delta_{O_{k}}^{(v)}-1\right)$ and $I_{k}\left(\Delta_{O_{k}}^{(v)}\right)$, i.e., the $I / S$ before and after admitting the new call, respectively. From (6), $I_{k}\left(\Delta_{O_{k}}^{(v)}\right)$ is the sum of random variables of the form

$$
X_{i k}^{(j)}=\frac{D^{4}\left(U_{j i}^{v}, B_{i}\right) 10^{-\frac{\psi_{j i}^{v}}{10}}}{D^{4}\left(U_{j i}^{v}, B_{k}\right) 10^{-\frac{\psi_{j k}^{v}}{10}}} .
$$

The number of such random variables is equal to the number of other cell interferers $\Delta_{O_{k}}^{(v)}$. For large values of $\Delta_{O_{k}}^{(v)}$, CLT can be applied to approximate $I_{k}\left(\Delta_{O_{k}}^{(v)}\right)$ to be a normally distributed random variable with mean equal to the sum of the means of the individual random variables $X_{i k}^{(j)}$, and variance equal to the sum of the variances of the $X_{i k}^{(j)}$. The mean and the variance of $X_{i k}^{(j)}$ can be obtained by noting that $X_{i k}^{(j)}$ is a lognormal random variable of the form $X_{i k}^{(j)}=e^{-a \Omega_{i k}^{(j)}}$, where $a=(\ln (10)) / 10$, $\Omega_{i k}^{(j)} \sim \mathcal{N}\left(\mu_{i k}^{(j)}, 2 \sigma^{2}\right)$, and $\mu_{i k}^{(j)}$ is given by

$$
\mu_{i k}^{(j)}=10 \log _{10} \frac{D^{4}\left(U_{j i}^{v}, B_{i}\right)}{D^{4}\left(U_{j i}^{v}, B_{k}\right)} .
$$

The mean and variance of $X_{i k}^{(j)}$ can then be obtained from the moment generating function of $\Omega_{i k}^{(j)}$, as

$$
E\left[X_{i k}^{(j)}\right]=e^{\left(-a \mu_{i k}^{(j)}+a^{2} \sigma^{2}\right)}
$$

and

$$
\operatorname{var}\left[X_{i k}^{(j)}\right]=e^{\left(-2 a \mu_{i k}^{(j)}+2 a^{2} \sigma^{2}\right)}\left(e^{2 a^{2} \sigma^{2}}-1\right) .
$$

Therefore by applying CLT, the mean $\mu_{\Delta_{O_{k}}^{(v)}}$, and variance $\sigma_{\Delta_{O_{k}}^{(v)}}^{2}$ of $I_{k}\left(\Delta_{O_{k}}^{(v)}\right)$ can be obtained as

$$
\begin{aligned}
\mu_{\Delta_{O_{k}}^{(v)}} & =\sum_{\substack{i \in S_{k} \\
i \neq k}} \sum_{j=1}^{\Delta_{i k}^{(v)}} e^{\left(-a \mu_{i k}^{(j)}+a^{2} \sigma^{2}\right)} \\
\sigma_{\Delta_{O_{k}}^{(v)}}^{2} & =\sum_{\substack{i \in S_{k} \\
i \neq k}} \sum_{j=1}^{\Delta_{i k}^{(v)}} e^{\left(-2 a \mu_{i k}^{(j)}+2 a^{2} \sigma^{2}\right)}\left(e^{2 a^{2} \sigma^{2}}-1\right)
\end{aligned}
$$

where $\sum_{\substack{i \in S_{k} \\ i \neq k}} \Delta_{i k}^{(v)}=\Delta_{O_{k}}^{(v)}$. Since the positions of the users are random, the means are random and are statistically independent of each other. The outage probability obtained is, therefore, conditioned on the positions of the users in the different cells and, hence, have to be averaged over the location of the interferers. We assume that the means $\mu_{i k}^{(j)}$ are independent an identically distributed (i.i.d) random variables, which is valid due to the symmetry of the system. Hence, (24) and (25) can be modified as

$$
\begin{aligned}
& \mu_{\Delta_{O_{k}}^{(v)}}=\Delta_{O_{k}}^{(v)} e^{\left(-a \mu_{i k}^{(j)}+a^{2} \sigma^{2}\right)} \\
& \sigma_{\Delta_{O_{k}}^{(v)}}^{2(v)}=\Delta_{O_{k}}^{(v)} e^{\left(-2 a \mu_{i k}^{(j)}+2 a^{2} \sigma^{2}\right)}\left(e^{\left.2 a^{2} \sigma^{2}-1\right) .}\right.
\end{aligned}
$$

Similarly, $I_{k}\left(\Delta_{O_{k}}^{(v)}-1\right)$ can be modeled as a normal random variable with mean $\mu_{\Delta_{O_{k}}^{(v)}-1}$ and variance $\sigma_{\Delta_{O_{k}}^{(v)}-1}^{2}$, which can be obtained from (26) and (27) by replacing $\Delta_{O_{k}}^{(v)}$ by $\Delta_{O_{k}}^{(v)}-1$. It is observed that $I_{k}\left(\Delta_{O_{k}}^{(v)}-1\right)$ and $I_{k}\left(\Delta_{O_{k}}^{(v)}\right)$ are jointly normal with a correlation coefficient $r$. If $f_{k}(x, y)$ is the joint probability density function (pdf) of $I_{k}\left(\Delta_{O_{k}}^{(v)}-1\right)$ and $I_{k}\left(\Delta_{O_{k}}^{(v)}\right)$, then

$$
f_{k}(x, y)=\frac{e^{-\frac{1}{2\left(1-r^{2}\right)}\left[\frac{\hat{\hat{x}}^{2}}{\sigma_{1}^{2}}-\frac{2 r \hat{\hat{\hat{y}}} \hat{y}}{\sigma_{1}}+\frac{\hat{\hat{y}}^{2}}{\sigma_{2}^{2}}\right]}}{2 \pi \sigma_{1} \sigma_{2} \sqrt{\left(1-r^{2}\right)}}
$$


where $\hat{x}=x-\mu_{\Delta_{O_{k}}^{(v)}}, \hat{y}=y-\mu_{\Delta_{O_{k}}^{(v)}-1}, \sigma_{1}=\sigma_{\Delta_{O_{k}}^{(v)}}$, and $\sigma_{2}=\sigma_{\Delta_{O_{k}}^{(v)}-1}$. The correlation coefficient $r$ is defined as

$$
r=\frac{E\left[I_{k}\left(\Delta_{O_{k}}^{(v)}-1\right) I_{k}\left(\Delta_{O_{k}}^{(v)}\right)\right]-\mu_{\Delta_{O_{k}}^{(v)}} \mu_{\Delta_{O_{k}}^{(v)}-1}}{\sigma_{\Delta_{O_{k}}^{(v)}} \sigma_{\Delta_{O_{k}}^{(v)}-1}} .
$$

To evaluate $E\left[I_{k}\left(\Delta_{O_{k}}^{(v)}-1\right) I_{k}\left(\Delta_{O_{k}}^{(v)}\right)\right]$ in (29), we rewrite $I_{k}\left(\Delta_{O_{k}}^{(v)}\right)$ in (6) as

$$
I_{k}\left(\Delta_{O_{k}}^{(v)}\right)=I_{k}\left(\Delta_{O_{k}}^{(v)}-1\right)+X_{i k}^{(j)}
$$

where $X_{i k}^{(j)}$, given by (20), denotes the additional interference caused by the newly admitted call which, without loss of generality, is from the $j$ th user in cell $i$. From (30) and (20), we can evaluate $E\left[I_{k}\left(\Delta_{O_{k}}^{(v)}\right) I_{k}\left(\Delta_{O_{k}}^{(v)}-1\right)\right]$ by using the fact that $X_{i k}^{(j)}$ and $I_{k}\left(\Delta_{O_{k}}^{(v)}-1\right)$ are statistically independent. Conditioned on the location of the newly admitted call, $X_{i k}^{(j)}$ is a log-normally distributed random variable of the form $X_{i k}^{(j)}=10^{-\Omega_{i k}^{(j)}} / 10$, where $\Omega_{i k}^{(j)} \sim \mathcal{N}\left(\mu_{i k}^{(j)}, 2 \sigma^{2}\right)$. Hence, by using the CLT approximation on $I_{k}\left(\Delta_{O_{k}}^{(v)}\right)$ and $I_{k}\left(\Delta_{O_{k}}^{(v)}-1\right)$, we can evaluate the joint probability in (18) from (20) and (28)-(30) as

$$
\begin{aligned}
\operatorname{Pr} & \left\{I_{k}\left(\Delta_{O_{k}}^{(v)}\right)>\epsilon_{v}^{\prime}, I_{k}\left(\Delta_{O_{k}}^{(v)}-1\right) \leq \epsilon_{v}^{\prime}\right\} \\
& =\left(\frac{1}{\pi R^{2}}\right) \iiint_{\epsilon_{v}^{\prime}}^{\infty} \int_{-\infty}^{\epsilon_{v}^{\prime}} f_{\Delta_{O_{k}}^{(v)}}(x, y) r_{1} d y d x d r_{1} d \theta_{1} .
\end{aligned}
$$

Approximation Using Fenton's Method: In this subsection, we present an approximation using Fenton's method [12] to evaluate the joint probability $P_{j}$ in (18). Each term in the summation in (6) is a log-normal random variable of the form $10^{-\xi / 10}$, where $\xi \sim \mathcal{N}\left(\mu_{i k}^{(j)}, 2 \sigma^{2}\right)$. Hence, by modeling the terms in the summation of (6) to be i.i.d, $I_{k}\left(\Delta_{O_{k}}^{(v)}-1\right)$ can be approximated to follow a log-normal distribution of the form $10^{-\Omega / 10}$, where $\Omega \sim \mathcal{N}\left(\hat{\mu}_{\Delta_{O_{k}}^{(v)}-1}, \hat{\sigma}_{\Delta_{O_{k}}^{(v)}-1}^{2}\right)$. We apply Fenton's method (i.e., approximate the sum of independent log-normal random variables by a log-normal random variable) to obtain the expressions for $\hat{\mu}_{\Delta_{O_{k}}^{(v)}-1}$ and $\hat{\sigma}_{\Delta_{O_{k}}^{(v)}-1}^{2}$, as

$$
\hat{\sigma}_{\Delta_{O_{k}}^{(v)}-1}^{2}=\frac{1}{a^{2}} \ln \left[1+\frac{e^{2 a^{2} \sigma^{2}}-1}{\Delta_{O_{k}}^{(v)}-1}\right]
$$

and

$$
\begin{aligned}
\hat{\mu}_{\Delta_{O_{k}}^{(v)}-1}= & \mu_{i k}^{(j)}+\frac{a}{2}\left(\sigma_{\Delta_{O_{k}}^{(v)}-1}^{2}-2 \sigma^{2}\right) \\
& -\frac{1}{a} \ln \left(\Delta_{O_{k}}^{(v)}-1\right) .
\end{aligned}
$$

We rewrite the joint probability in (18) as

$$
\operatorname{Pr}\left\{\tilde{I}_{k}\left(\Delta_{O_{k}}^{(v)}\right)>\epsilon_{d B}^{v^{\prime}}, \tilde{I}_{k}\left(\Delta_{O_{k}}^{(v)}-1\right) \leq \epsilon_{d B}^{v^{\prime}}\right\}
$$

where $\epsilon_{d B}^{v^{\prime}}=10 \log _{10}\left(\epsilon_{v}^{\prime}\right), \tilde{I}_{k}\left(\Delta_{O_{k}}^{(v)}\right)=10 \log _{10} I_{k}\left(\Delta_{O_{k}}^{(v)}\right)$ and $\tilde{I}_{k}\left(\Delta_{O_{k}}^{(v)}-1\right)=10 \log _{10} I_{k}\left(\Delta_{O_{k}}^{(v)}-1\right)$. Since $I_{k}\left(\Delta_{O_{k}}^{(v)}\right)$ and
$I_{k}\left(\Delta_{O_{k}}^{(v)}-1\right)$ are log-normal, $\tilde{I}_{k}\left(\Delta_{O_{k}}^{(v)}-1\right)$ and $\tilde{I}_{k}\left(\Delta_{O_{k}}^{(v)}\right)$ are jointly normal with correlation coefficient $\hat{r}$, given by

$$
\hat{r}=\frac{E\left[\tilde{I}\left(\Delta_{O_{k}}^{(v)}-1\right) \tilde{I}\left(\Delta_{O_{k}}^{(v)}\right)\right]-\hat{\mu}_{\Delta_{O_{k}}^{(v)}-1} \hat{\mu}_{\Delta_{O_{k}}^{(v)}}}{\hat{\sigma}_{\Delta_{O_{k}}^{(v)}-1} \hat{\sigma}_{\Delta_{O_{k}}^{(v)}}} .
$$

To evaluate the expectation $E\left[\tilde{I}_{k}\left(\Delta_{O_{k}}^{(v)}-1\right) \tilde{I}_{k}\left(\Delta_{O_{k}}^{(v)}\right)\right]$, we use (30) and use $\ln (1+x) \approx x$ for $|x|<1$, to obtain

$$
\tilde{I}_{k}\left(\Delta_{O_{k}}^{(v)}\right)=\tilde{I}_{k}\left(\Delta_{O_{k}}^{(v)}-1\right)+\frac{X_{i k}^{(j)}}{I_{k}\left(\Delta_{O_{k}}^{(v)}-1\right)}
$$

From (36), we obtain

$$
\begin{gathered}
E\left[\tilde{I}_{k}\left(\Delta_{O_{k}}^{(v)}-1\right) \tilde{I}_{k}\left(\Delta_{O_{k}}^{(v)}\right)\right]=E\left[\tilde{I}_{k}^{2}\left(\Delta_{O_{k}}^{(v)}-1\right)\right] \\
+E\left[\tilde{I}_{k}\left(\Delta_{O_{k}}^{(v)}-1\right) \frac{X_{i k}^{(j)}}{I_{k}\left(\Delta_{O_{k}}^{(v)}-1\right)}\right] .
\end{gathered}
$$

Using $\ln (1+x) \approx x$ for $|x|<1$ again, we have

$$
\begin{aligned}
& E\left[\tilde{I}_{k}\left(\Delta_{O_{k}}^{(v)}-1\right) \tilde{I}_{k}\left(\Delta_{O_{k}}^{(v)}\right)\right] \\
& =E\left[\tilde{I}_{k}^{2}\left(\Delta_{O_{k}}^{(v)}-1\right)\right]+\frac{1}{a^{2}} E\left[\frac{X_{i k}^{(j)}}{I_{k}\left(\Delta_{O_{k}}^{(v)}-1\right)}\right] \\
& \quad-\frac{1}{a^{2}} E\left[\frac{X_{i k}^{(j)}}{I_{k}^{2}\left(\Delta_{O_{k}}^{(v)}-1\right)}\right] .
\end{aligned}
$$

The ratios $X_{i k}^{(j)} /\left(I_{k}\left(\Delta_{O_{k}}^{(v)}\right)-1\right)$ and $X_{i k}^{(j)} /\left(I_{k}^{2}\left(\Delta_{O_{k}}^{(v)}-1\right)\right)$ are log-normal variables of the form $10^{-(\hat{\Omega} / 10)}$ and $10^{-(\tilde{\Omega} / 10)}$, respectively, where $\hat{\Omega} \sim \mathcal{N}\left(\mu_{i k}^{(j)}-\hat{\mu}_{\Delta_{O_{k}}^{(v)}-1}, 2 \sigma^{2}+\hat{\sigma}_{\Delta_{O_{k}}^{(v)}-1}^{2}\right)$, and $\tilde{\Omega} \sim \mathcal{N}\left(\mu_{i k}^{(j)}-2 \hat{\mu}_{\Delta_{O_{k}}^{(v)}-1}, 2 \sigma^{2}+2 \hat{\sigma}_{\Delta_{O_{k}}^{(v)}-1}^{2}\right)$. From (35) and (38), we obtain (34) as

$$
\begin{aligned}
& \operatorname{Pr}\left\{\tilde{I}_{k}\left(\Delta_{O_{k}}^{(v)}\right)>\epsilon_{d B}^{v^{\prime}}, \tilde{I}_{k}\left(\Delta_{O_{k}}^{(v)}-1\right) \leq \epsilon_{d B}^{v^{\prime}}\right\} \\
& =\left(\frac{1}{\pi R^{2}}\right) \int_{\epsilon_{d B}^{v^{\prime}}}^{\infty} \int_{-\infty}^{\epsilon_{d B}^{v^{\prime}}} \iint \tilde{f}_{\Delta_{O_{k}}^{(v)}}(x, y) r_{1} d y d x d r_{1} d \theta_{1}
\end{aligned}
$$

where defining $\hat{x} \triangleq x-\hat{\mu}_{\Delta_{O_{k}}^{(v)}}$ and $\hat{y} \triangleq y-\hat{\mu}_{\Delta_{O_{k}}^{(v)}-1}, \tilde{f}_{\Delta_{O_{k}}^{(v)}}(x, y)$ is given by

$$
\tilde{f}_{\Delta_{O_{k}}^{(v)}}(x, y)=\frac{e^{-\frac{1}{2\left(1-\hat{r}^{2}\right)}\left[\frac{\hat{x}^{2}}{\hat{\sigma}_{1}^{2}}-\frac{2 \hat{r} \hat{x} \hat{y}}{\hat{\sigma}_{1} \hat{\sigma}_{2}}+\frac{\hat{y}^{2}}{\hat{\sigma}_{2}^{2}}\right]}}{2 \pi \hat{\sigma}_{1} \hat{\sigma}_{2} \sqrt{\left(1-\hat{r}^{2}\right)}} .
$$

In the above, $\hat{\sigma}_{1}=\hat{\sigma}_{\Delta_{O_{k}}^{(v)}}$ and $\hat{\sigma}_{2}=\hat{\sigma}_{\Delta_{O_{k}}^{(v)}-1}$. From (39), we can evaluate (34) and, hence, (18).

\section{B. Mixed Voice/Data System}

In this subsection, we derive analytical expressions for the performance measures of interest in a cellular CDMA system with mixed voice/data traffic employing SIR-based admission 
control. The parameters of interest are the outage probability for voice traffic, the average system throughput, and the mean delay for data traffic. We first derive the data burst retransmission probability which is then used to derive the average system throughput and the mean data burst delay.

1) Data Burst Retransmission Probability: A data burst currently in transmission could be lost because of a new voice call or a data burst being admitted into the system. Such a lost data burst is assumed to be regenerated after a random time and retransmitted. Let $p_{o}^{v}$ and $p_{o}^{d}$ denote the probabilities of data burst retransmission in cell $k$ due to a newly admitted voice call and data burst, respectively, in cell $i$. These probabilities conditioned on $\Delta_{O_{k}}^{(v)}, \Delta_{k}^{(d)}$, and $\Delta_{I_{k}}^{(v)}$ are denoted by $P_{v d}$ and $P_{d d}$, respectively. $\stackrel{P}{v d}_{\text {can }}$ be written as

$$
P_{v d}=\operatorname{Pr}\left\{I_{k}\left(\Delta_{O_{k}}^{(v)}, \Delta_{k}^{(d)}\right)>\epsilon_{d}^{\prime} \mid I_{k}\left(\Delta_{O_{k}}^{(v)}-1, \Delta_{k}^{(d)}\right) \leq \epsilon_{d}^{\prime}\right\}
$$

where $I_{k}\left(\Delta_{O_{k}}^{(v)}, \Delta_{k}^{(d)}\right)$ is obtained from (4) and $\epsilon_{d}^{\prime}=$ $\epsilon_{d}-\left(1 / k_{d}\right) \Delta_{I_{k}}^{(v)}$. Similarly, $P_{d d}$ can be written as

$P_{d d}=\operatorname{Pr}\left\{I_{k}\left(\Delta_{O_{k}}^{(v)}, \Delta_{k}^{(d)}\right)>\epsilon_{d}^{\prime} \mid I_{k}\left(\Delta_{O_{k}}^{(v)}, \Delta_{k}^{(d)}-1\right) \leq \epsilon_{d}^{\prime}\right\}$.

Averaging (41) and (42) over $\Delta_{O_{k}}^{(v)}$ and $\Delta_{k}^{(d)}$, we can write

$$
\begin{aligned}
& p_{o}^{v}\left(\Delta_{I_{k}}^{(v)}\right)=1-\prod_{\substack{i \in S_{k} \\
i \neq k}}\left[1-\sum_{M_{v}} \sum_{M_{d}} P_{v d} \operatorname{Pr}\left\{\Delta_{O_{i}}^{(v)}=M_{v}\right\}\right. \\
& \left.\operatorname{Pr}\left\{\Delta_{i}^{(d)}=M_{d}\right\}\right] \\
& p_{o}^{d}\left(\Delta_{I_{k}}^{(v)}\right)=1-\prod_{\substack{i \in S_{k} \\
i \neq k}}\left[1-\sum_{M_{v}} \sum_{M_{d}} P_{d d} \operatorname{Pr}\left\{\Delta_{O_{i}}^{(v)}=M_{v}\right\}\right. \\
& \left.. \operatorname{Pr}\left\{\Delta_{i}^{(d)}=M_{d}\right\}\right]
\end{aligned}
$$

where $\operatorname{Pr}\left\{\Delta_{O_{i}}^{(v)}=M_{v}\right\}$ and $\operatorname{Pr}\left\{\Delta_{k}^{(d)}=M_{d}\right\}$, based on similar arguments in Section III-A, are given by (10) and

$$
\operatorname{Pr}\left\{\Delta_{k}^{(d)}=M_{d}\right\}=\frac{e^{-\hat{\rho}_{d}} \hat{\rho}_{d}^{M_{d}}}{M_{d} !}
$$

respectively. In (45), $\hat{\rho}_{d}=\left(N_{k}+1\right) \rho_{d}$. Averaging (43) and (44) over $\Delta_{I_{k}}^{(v)}$, we have

$$
\begin{aligned}
& p_{o}^{v}=\sum_{m_{v}} p_{o}^{v}\left(\Delta_{I_{k}}^{(v)}\right) \operatorname{Pr}\left\{\Delta_{I_{k}}^{(v)}=m_{v}\right\} \\
& p_{o}^{d}=\sum_{m_{v}} p_{o}^{d}\left(\Delta_{I_{k}}^{(v)}\right) \operatorname{Pr}\left\{\Delta_{I_{k}}^{(v)}=m_{v}\right\}
\end{aligned}
$$

where $\operatorname{Pr}\left\{\Delta_{I_{k}}^{(v)}=m_{v}\right\}$ is as given in (12). The data burst retransmission probability $p_{o}$ is then given by

$$
p_{o}=\frac{\lambda_{v} p_{o}^{v}+\lambda_{d} p_{o}^{d}}{\lambda_{v}+\lambda_{d}} .
$$

In order to evaluate (41) and (42), we need to compute the joint probabilities

$$
\begin{aligned}
P_{v}^{J} \triangleq & \operatorname{Pr}\left\{I_{k}\left(\Delta_{O_{k}}^{(v)}, \Delta_{k}^{(d)}\right)>\epsilon_{d}^{\prime},\right. \\
\left.I_{k}\left(\Delta_{O_{k}}^{(v)}-1, \Delta_{k}^{(d)}\right) \leq \epsilon_{d}^{\prime}\right\} & \\
P_{d}^{J} \triangleq \operatorname{Pr}\{ & I_{k}\left(\Delta_{k}^{(v)}, \Delta_{k}^{(d)}\right)>\epsilon_{d}^{\prime}, \\
& \left.I_{k}\left(\Delta_{k}^{(v)}, \Delta_{k}^{(d)}-1\right) \leq \epsilon_{d}^{\prime}\right\}
\end{aligned}
$$

and the marginal probabilities

$$
\begin{aligned}
& P_{v}^{M} \triangleq \operatorname{Pr}\left\{I_{k}\left(\Delta_{O_{k}}^{(v)}-1, \Delta_{k}^{(d)}\right) \leq \epsilon_{d}^{\prime}\right\} \\
& P_{d}^{M} \triangleq \operatorname{Pr}\left\{I_{k}\left(\Delta_{O_{k}}^{(v)}, \Delta_{k}^{(d)}-1\right) \leq \epsilon_{d}^{\prime}\right\} .
\end{aligned}
$$

Note that $P_{v}^{J}, P_{v}^{M}, P_{d}^{J}$, and $P_{d}^{M}$ are similar to the expressions in (18) and (19). They can be evaluated by the methods discussed in Section III-A2. However, it will be seen later in Fig. 2, in Section IV, that the Fenton's method approximation to evaluate $P_{j}$ (i.e., (39)) together with the CB approximation to evaluate $P_{m}$ gives results closest to those obtained by simulations. Therefore, we apply the CB approximation in [2] to evaluate the marginal probabilities $P_{v}^{M}$ and $P_{d}^{M}$. To evaluate the joint probabilities $P_{v}^{J}$ and $P_{d}^{J}$, we apply the Fenton's method as explained in Section III-A2. We substitute the values of $P_{v}^{J}, P_{v}^{M}, P_{d}^{J}$, and $P_{d}^{M}$ in (43), (44), and (46)-(48) to obtain the data burst retransmission probability $p_{o}$.

The voice call outage probability due to a newly admitted voice call is obtained by scaling (41) by the factor $k_{d}$ and the voice call outage probability due to a newly admitted data burst is obtained by scaling (42) by $k_{d}$. Hence, the data burst retransmission probability, $p_{o}$, is also equal to the voice call outage probability. Also, the probability that a voice call is blocked or that a data burst is not admitted due to $I / S$ constraint and, hence, buffered $p_{b}$, can be written as

$$
p_{b}=\operatorname{Pr}\left\{I_{k}\left(\Delta_{k}^{(v)}, \Delta_{k}^{(d)}\right)>\epsilon_{d}\right\}
$$

We define the average system throughput, $\eta$, to be the fraction of time during which the system carries voice traffic and successful data bursts, which is given by

$$
\eta=\frac{\rho_{v} r^{(v)}\left(1-p_{b}\right)+\rho_{d} r^{(d)}\left(1-p_{b}\right)\left(1-p_{o}\right)}{n r^{(d)}} .
$$

2) Mean Data Burst Delay: In this subsection, we present the analysis for deriving the mean data burst delay. The data bursts in the virtual buffer wait till $a$ ) a code is available for allocation, and $b$ ) the $I / S$ at the corresponding base station is below threshold. We assume that the probability of a code not being available is small for the loads under consideration. Hence, the first departing data burst waits till the $I / S$ at its corresponding base station (in this case, base station $k$ ) goes below threshold. This happens only if an ongoing call departs from the system.

We define $p_{f}\left(\Delta_{k}^{(v)}, \Delta_{k}^{(d)}\right)$ to be the probability that the $I / S$ at the base station of cell $k$ goes below threshold following the 
departure of either a voice call or a data burst, which can be written as

$$
\begin{aligned}
p_{f}\left(\Delta_{k}^{(v)}, \Delta_{k}^{(d)}\right)= & p_{f}^{v}\left(\Delta_{k}^{(v)}, \Delta_{k}^{(d)}\right) p_{d}^{v}\left(\Delta_{k}^{(v)}, \Delta_{k}^{(d)}\right) \\
& +p_{f}^{d}\left(\Delta_{k}^{(v)}, \Delta_{k}^{(d)}\right) p_{d}^{d}\left(\Delta_{k}^{(v)}, \Delta_{k}^{(d)}\right)
\end{aligned}
$$

where $p_{f}^{v}\left(\Delta_{k}^{(v)}, \Delta_{k}^{(d)}\right)$ and $p_{f}^{d}\left(\Delta_{k}^{(v)}, \Delta_{k}^{(d)}\right)$ are the probabilities that the $I / S$ going below threshold is due to the departure of a voice call and a data burst, respectively. Likewise, $p_{d}^{v}\left(\Delta_{k}^{(v)}, \Delta_{k}^{(d)}\right)$ and $p_{d}^{d}\left(\Delta_{k}^{(v)}, \Delta_{k}^{(d)}\right)$ are the probabilities that the departing call is a voice call and a data burst, respectively. $p_{f}^{v}\left(\Delta_{k}^{(v)}, \Delta_{k}^{(d)}\right)$ and $p_{f}^{d}\left(\Delta_{k}^{(v)}, \Delta_{k}^{(d)}\right)$ are given by

$$
\begin{aligned}
& p_{f}^{v}\left(\Delta_{k}^{(v)}, \Delta_{k}^{(d)}\right)=\operatorname{Pr}\{ I_{k}\left(\Delta_{O_{k}}^{(v)}-1, \Delta_{k}^{(d)}\right) \leq \epsilon_{d}^{\prime} \\
&\left.\mid I_{k}\left(\Delta_{O_{k}}^{(v)}, \Delta_{k}^{(d)}\right)>\epsilon_{d}^{\prime}\right\} \\
& p_{f}^{d}\left(\Delta_{O_{k}}^{(v)}, \Delta_{k}^{(d)}\right)=\operatorname{Pr}\left\{I_{k}\left(\Delta_{O_{k}}^{(v)}, \Delta_{k}^{(d)}-1\right) \leq \epsilon_{d}^{\prime}\right. \\
& \\
&\left.\mid I_{k}\left(\Delta_{O_{k}}^{(v)}, \Delta_{k}^{(d)}\right)>\epsilon_{d}^{\prime}\right\} .
\end{aligned}
$$

The above two equations are evaluated by the method applied to evaluate $p_{o}^{v}$ and $p_{o}^{d}$ in Section III-B1. The probabilities $p_{d}^{v}\left(\Delta_{O_{k}}^{(v)}, \Delta_{k}^{(d)}\right)$ and $p_{d}^{d}\left(\Delta_{O_{k}}^{(v)}, \Delta_{k}^{(d)}\right)$ are given by

$$
\begin{aligned}
& p_{d}^{v}\left(\Delta_{k}^{(v)}, \Delta_{k}^{(d)}\right)=\frac{\Delta_{k}^{(v)} \mu_{v}}{\Delta_{k}^{(v)} \mu_{v}+\Delta_{k}^{(d)} \mu_{d}} \\
& p_{d}^{d}\left(\Delta_{k}^{(v)}, \Delta_{k}^{(d)}\right)=\frac{\Delta_{k}^{(d)} \mu_{d}}{\Delta_{k}^{(v)} \mu_{v}+\Delta_{k}^{(d)} \mu_{d}} .
\end{aligned}
$$

The arrival rate into the virtual queue is $N \lambda_{d}$, where $N=61$ is the total number of cells in the system. Let $m$ be the number of departures that need to occur for the $I / S$ at the base station of cell $k$ to go below threshold. Let $T$ be the random variable that denotes the delay experienced by the first departing data burst in the virtual queue. Let $T_{i}, i \in\{1,2, \cdots, m\}, T_{m}=T$, be the epoch at which the $i$ th departure occurs. Let $Y_{i} \triangleq T_{i}-T_{i-1}$, $i \in\{1,2, \cdots, m\}, T_{0}=0$. Hence, the delay $T$ of the first departing data burst is given by

$$
T=\sum_{i=1}^{m} Y_{i}
$$

It is noted that the random variables $Y_{i}$ are independent of each other, i.e., $Y_{i} \amalg Y_{j}, i \neq j$. The characteristic function of $T$ conditioned on $m, \Delta_{k}^{(v)}$, and $\Delta_{k}^{(d)}, \phi_{T}\left(\omega \mid m, \Delta_{k}^{(v)}, \Delta_{k}^{(d)}\right)$, is given by

$$
\phi_{T}\left(\omega \mid m, \Delta_{k}^{(v)}, \Delta_{k}^{(d)}\right)=\prod_{l=1}^{m} \phi_{l}(j \omega)
$$

where $\phi_{l}\left(\omega \mid K, \Delta_{k}^{(v)}, \Delta_{k}^{(d)}\right)$, the characteristic function of $Y_{l}$ conditioned on $m, \Delta_{k}^{(v)}$ and $\Delta_{k}^{(d)}$, is given by

$$
\phi_{l}(j \omega)=\sum_{i=1}^{l}\left[\frac{\mu_{l}^{i}}{\mu_{l}^{i}-j \omega}\right]\left(\begin{array}{l}
l-1 \\
i-1
\end{array}\right) \cdot\left(p_{d}^{v}\right)^{l-i} \cdot\left(p_{d}^{d}\right)^{i-1}
$$

In the above, $\mu_{l}^{i}$ is given by

$$
\mu_{l}^{i}=\left[\Delta_{k}^{(v)}-(l-i)\right] \mu_{v}+\left[\Delta_{k}^{(d)}-(i-1)\right] \mu_{d} .
$$

Equation (62) is obtained from the fact that conditioned on the type of each departing call, $Y_{i}$ is an exponentially distributed random variable. $p_{d}^{v}$ and $p_{d}^{d}$ are obtained by averaging (58) and (59) over $\Delta_{k}^{(v)}$ and $\Delta_{k}^{(d)}$. Note that the averaging over $\Delta_{k}^{(v)}$ and $\Delta_{k}^{(d)}$ should not be done at this stage and needs to be done on the expression for the mean delay of the first departing data burst conditioned on $\Delta_{k}^{(v)}$ and $\Delta_{k}^{(d)}$. However, we have averaged (58) and (59) at this stage to simplify our analysis. This can be valid for low loads because the probability of a buffered data burst having to wait for more than one departure for the $I / S$ go below threshold is quite small. It can also be valid for high loads because the difference in the ratios in the right-hand side (RHS) of (58) and (59) is negligible for changes of the order of unity in $\Delta_{k}^{(v)}$ and $\Delta_{k}^{(d)}$.

To average (61) over $m$, we use

$$
\operatorname{Pr}\{m=K\}=\sum_{j=1}^{K} P_{a}\left(\begin{array}{c}
K \\
j
\end{array}\right)\left(p_{d}^{v}\right)^{j}\left(p_{d}^{d}\right)^{K-j}
$$

where

$$
\begin{aligned}
P_{a}= & \prod_{l=0}^{j-1}\left[1-p_{f}\left(\Delta_{k}^{(v)}-l, \Delta_{k}^{(d)}-(j-l)\right)\right] \\
& \times p_{f}\left(\Delta_{k}^{(v)}-j, \Delta_{k}^{(d)}-(K-j)\right) .
\end{aligned}
$$

Equation (64) is further simplified by replacing $p_{f}\left(\Delta_{k}^{(v)}-\right.$ $\left.l, \Delta_{k}^{(d)}-(j-l)\right)$ by $p_{f}$, where $p_{f}$ is obtained by averaging $p_{f}\left(\Delta_{k}^{(v)}, \Delta_{k}^{(d)}\right)$ over $\Delta_{k}^{(v)}$ and $\Delta_{k}^{(d)}$. It is noted again that the averaging over $\Delta_{k}^{(v)}$ and $\Delta_{k}^{(d)}$ must not be done at this stage. However, we make this approximation to simplify the analysis with the same justification as mentioned earlier. By making this approximation, (64) is modified as

$$
\operatorname{Pr}\{m=K\}=\left(1-p_{f}\right)^{K-1} p_{f} .
$$

Averaging (61) over $m, \Delta_{k}^{(v)}$ and $\Delta_{k}^{(d)}$, we obtain the characteristic function of $T, \phi_{T}(\omega)$. The density function of the delay $T, f_{T}(t)$, is then given by

$$
f_{T}(t)=\frac{1}{2 \pi} \int_{-\infty}^{\infty} \phi_{T}(\omega) e^{-j \omega t} d \omega
$$

The mean delay $T_{\text {ave }}$ and the delay variance $T_{\text {var }}$ of the first departing data burst are given by

$$
T_{\text {ave }}=\int_{t} t f_{T}(t) d t
$$

and

$$
T_{\mathrm{var}}=\int_{t}\left(t-T_{\mathrm{ave}}\right)^{2} f_{T}(t) d t
$$

The rest of the virtual queue, other than the first departing data burst, is modeled as an $M / G / 1$ queue with mean service time 


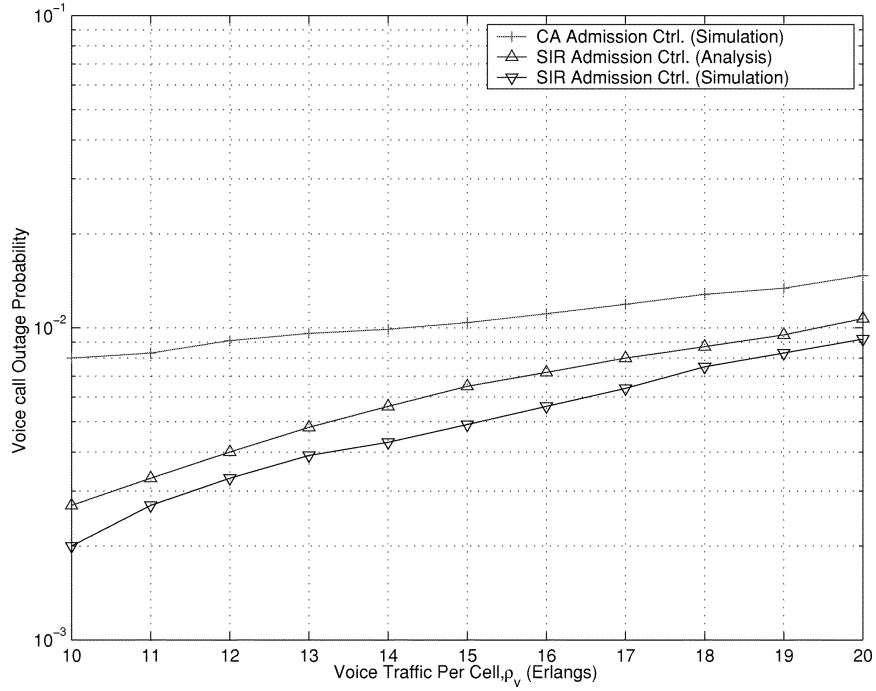

Fig. 1. Outage performance with SIR-based admission control and CA-based admission control. Voice-only system. $N=61$ cells. $n=64$ spreading codes.

$T_{\text {ave. }}$ Hence, we can obtain the mean waiting time $W_{\text {ave }}$ in the $M / G / 1$ queue, using the Pollackzek-Kinchine formula [11], as

$$
W_{\mathrm{ave}}=\frac{\rho T_{\mathrm{ave}}}{2(1-\rho)}\left(1+c_{T}^{2}\right)
$$

where $c_{T}^{2}=T_{\mathrm{var}} / T_{\mathrm{ave}}^{2}$ and $\rho=p_{b} N \lambda_{d} T_{\mathrm{ave}}$. Finally, the mean data burst delay $\bar{D}_{d}$ is given by

$$
\bar{D}_{d}=p_{b}\left(W_{\text {ave }}+T_{\text {ave }}\right) N_{t x}
$$

where $N_{t x}$ is the average number of transmissions per packet, given by

$$
N_{t x}=\frac{1}{1-p_{o}} .
$$

\section{RESULTS AND DISCUSSION}

In this section, we present the analytical and simulation results of the performance of a voice/data CDMA system with SIR-based admission control. The performance of the system with CA-based admission control is also presented for comparison. Our focus in the performance results and discussion are two fold. First, we compare the accuracy of the performance of the SIR-based admission control predicted by our approximate analysis with that obtained by actual simulations without any approximations. Second, we compare the performance gain achieved by using SIR-based admission control relative to that achieved by CA-based admission control. The latter is done by comparing the results obtained by actual simulations without any approximation. Consequently, in all the legends in Figs. 1-6, 'Simulation" means true simulations of the system without approximation.

The following system parameter values are used in all the analytical computations and simulations: $N=61$ cells, $n=64$ spreading codes, $\mu_{v}^{-1}=100 \mathrm{~s}, \mu_{d}^{-1}=1 \mathrm{~s}, r^{(v)}=8 \mathrm{~kb} / \mathrm{s}$, $r^{(d)}=16 \mathrm{~kb} / \mathrm{s}$ (i.e., $k_{d}=2$ ), and $\sigma=8 \mathrm{~dB}$. As in [4], we take $\epsilon_{v}=14 \mathrm{~dB}$ and, hence, $\epsilon_{d}=11 \mathrm{~dB}$.

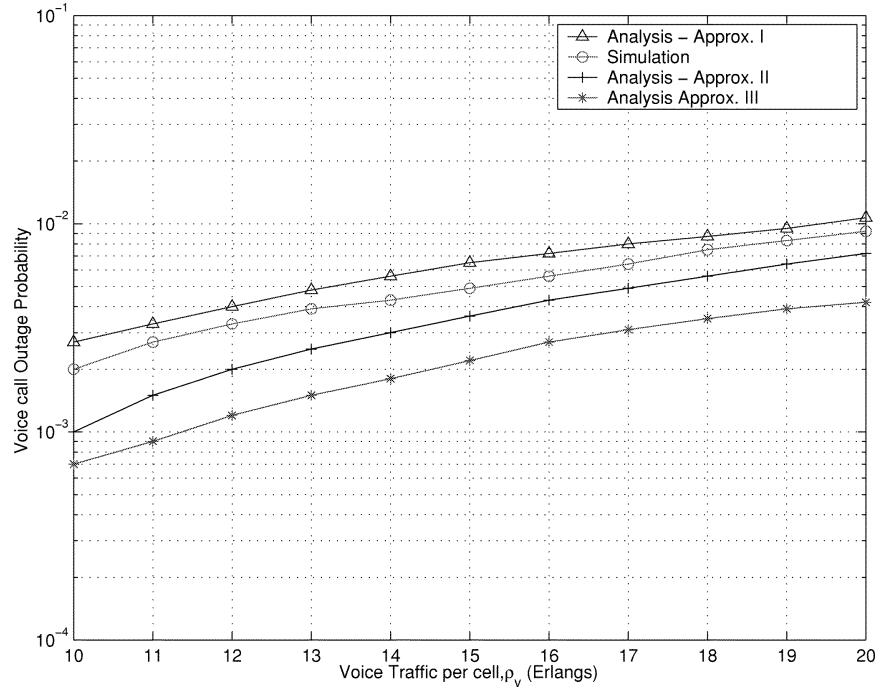

Fig. 2. Outage performance with SIR-based admission control predicted by analysis and simulations. Voice-only system. $N=61$ cells. $n=64$ spreading codes.

\section{A. Voice-Only System}

We first evaluate the outage probability for a voice-only system with and without SIR-based admission control. Fig. 1 shows the outage probability as a function of Erlang load per cell with both CA-based, as well as SIR-based admission control. The analytical results for the SIR-based admission control is obtained by using Fenton's method approximation to compute the joint probability $P_{j}$ [i.e., (39)] and by using the $\mathrm{CB}$ approximation given in [2] to evaluate the marginal probability, $P_{m}$. It is observed that with CA-based admission control, an outage probability of 0.01 occurs at a load of about $\rho_{v}=15$ Erlangs/cell, whereas with SIR-based admission control, the same outage performance is achieved at a load of $\rho_{v}=20$ Erlangs/cell. This is an increase of $33 \%$ in the Erlang capacity in a system with SIR-based admission control compared with a system with CA-based admission control. This improvement in capacity is due to admitting new calls only when $I / S$ criterion is not violated.

The accuracy of the outage performance predicted by the analysis discussed in Section III are illustrated in Fig. 2, by comparing the analytical results obtained through different approximations with the simulation results. The curve labeled "Approx. I" in Fig. 2 is obtained by computing $P_{j}$ through Fenton's method approximation [i.e., (39)] and $P_{m}$ through CB approximation as in [2]. The curve labeled "Approx. II" is obtained by computing $P_{j}$ through Fenton's method approximation [i.e., (39)] and $P_{m}$ through Edgeworth expansion as in [4]. The curve labeled "Approx. III" is obtained by computing $P_{j}$ through CLT [i.e., (31)] and $P_{m}$ through CLT approximation as in [2]. It is observed that the analytical results obtained through "Approx. I" overestimates the outage probability, whereas the results obtained through "Approx. II" and "Approx. III" underestimate it. From Fig. 2, we observe that the CB approximation for $P_{m}$ and Fenton's approximation for $P_{j}$ ("Approx. I") is more accurate (i.e., closer to the simulation curve) than the other approximations. 


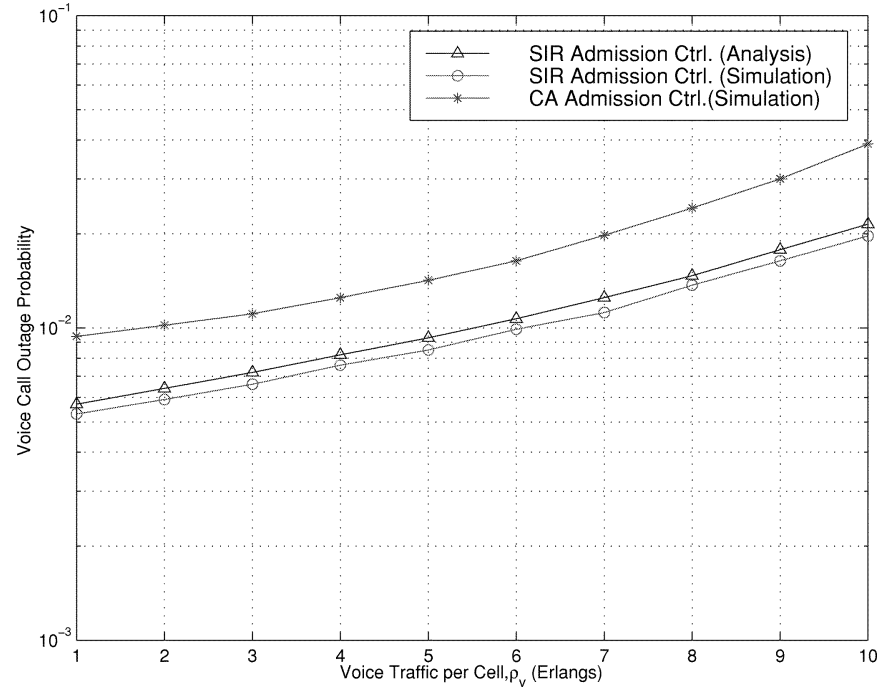

Fig. 3. Voice call outage performance as a function of voice traffic load $\rho_{v}$ with SIR-based and CA-based admission control. Mixed voice/data system. $\rho_{d}=5$ Erlangs per cell.

\section{B. Mixed Voice/Data System}

Next, we evaluate the performance of the mixed voice/data system with and without SIR-based admission control. We are interested in comparing the mean data burst delay performance and the voice/data Erlang capacities with SIR-based and CA-based admission control. We define the voice and data Erlang capacities as the offered voice traffic for a desired voice call outage probability and the offered data traffic for a desired mean data burst delay performance, respectively. We specifically consider a data-only system (for which $\rho_{v}=0$ ), as well as a mixed voice/data system with $\rho_{v}=10$ Erlangs per cell, both with varying $\rho_{d}$.

As observed in Section IV-A, "Approx. I" (i.e., evaluating $P_{m}$ using the $\mathrm{CB}$ approximation as in [2] and $P_{j}$ using the Fenton's method approximation [i.e., (39)]) matched best with the results obtained by simulations. Henceforth, in the subsequent figures, Figs. 3-6, the plots corresponding to the legend "SIR Admission Ctrl. (Analysis)" are obtained by evaluating $P_{v}^{M}$ and $P_{d}^{M}$ using the CB approximation as in [2] and evaluating $P_{v}^{J}$ and $P_{d}^{J}$ using the Fenton's method approximation explained in Section III-B.

Fig. 3 gives the voice call outage probability performance as a function of voice traffic load $\rho_{v}$ in a mixed voice/data system with a data traffic of $\rho_{d}=5$ Erlangs per cell. In Fig. 3, the curve labeled "SIR Admission Ctrl. (Analysis)" is obtained by evaluating (48). It is observed that the results obtained through our analysis closely match the simulation results. Also, the SIR-based admission control is seen to perform better than the CA-based admission control. For example, a 1\% outage probability occurs at a voice traffic of about 2 Erlangs per cell using CA-based admission control, whereas for the same outage performance of $1 \%$, the SIR-based admission control supports an increased voice traffic of about 6 Erlangs per cell. It is noted that, in the voice-only system that we studied, a voice traffic load of about 20 Erlangs per cell was achieved at a $1 \%$ voice call outage probability. However, in the mixed voice/data system, the voice Erlang capacity achieved is 6 Erlangs per cell in the presence of 5 Erlangs per cell of data traffic. Thus,

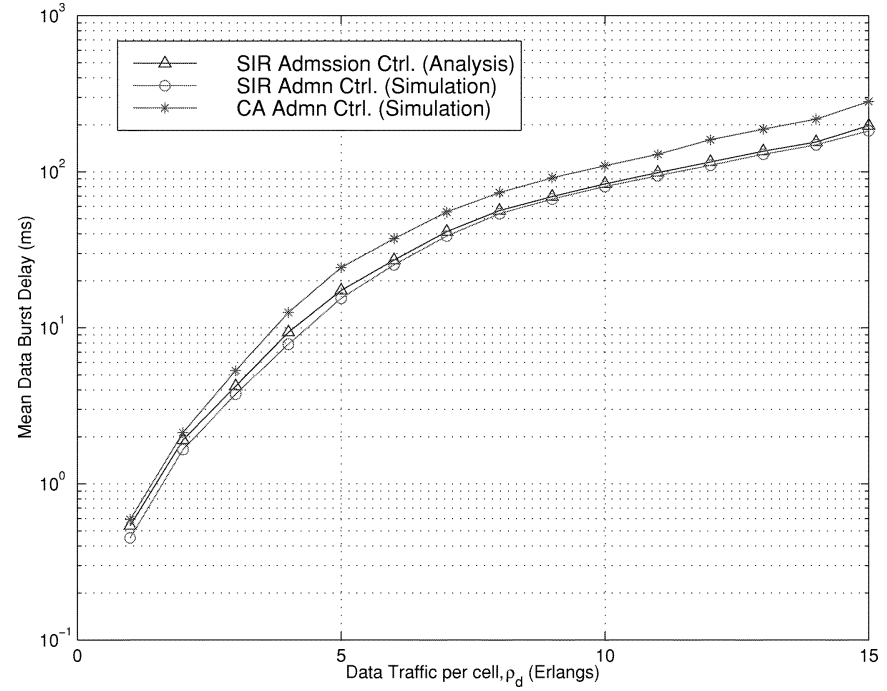

Fig. 4. Mean data burst delay $\bar{D}_{d}$ versus $\rho_{d}$ in the absence of voice traffic (i.e., $\left.\rho_{v}=0\right)$.

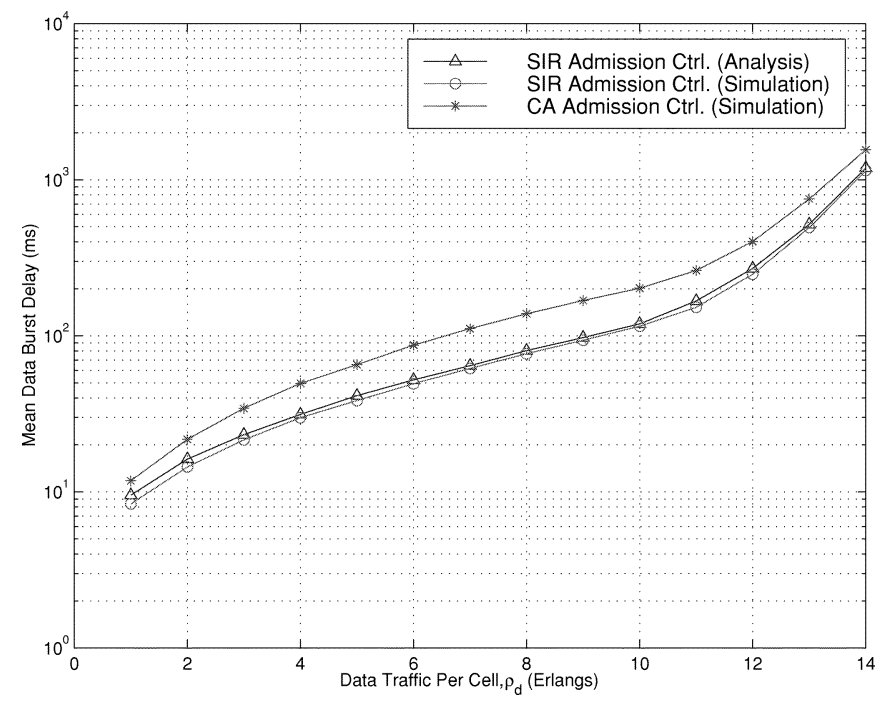

Fig. 5. Mean data burst delay $\bar{D}_{d}$ versus $\rho_{d}$ for $\rho_{v}=10$ Erlangs per cell.

the voice Erlang capacity comes down while supporting higher rate data users.

In Figs. 4 and 5, we compare the mean data burst delay performance of the SIR-based admission control with that of the CA-based admission control, as a function of data traffic $\rho_{d}$. Fig. 4 corresponds to a data-only system (i.e., $\rho_{v}=0$ ) and Fig. 5 corresponds to a mixed voice/data system with a voice traffic of $\rho_{v}=10$ Erlangs per cell. In Figs. 4 and 5, the curves labeled "SIR Admission Ctrl. (Analysis)" is obtained by evaluating (71). Again, the analytical mean delay results are observed to be close to the simulation results, thus validating the approximations made in the analysis. From Fig. 4, we observe that, in the absence of voice traffic, we obtain an improvement of about $25 \%$ in the mean delay performance due to SIR-based admission control compared with CA-based admission control (about $70 \mathrm{~ms}$ mean delay for CA-based admission control and about $53 \mathrm{~ms}$ mean delay for SIR-based admission control, at $\rho_{d}=8$ Erlangs per cell). This is because, in CA-based admission control, calls are admitted into the system regardless of the 


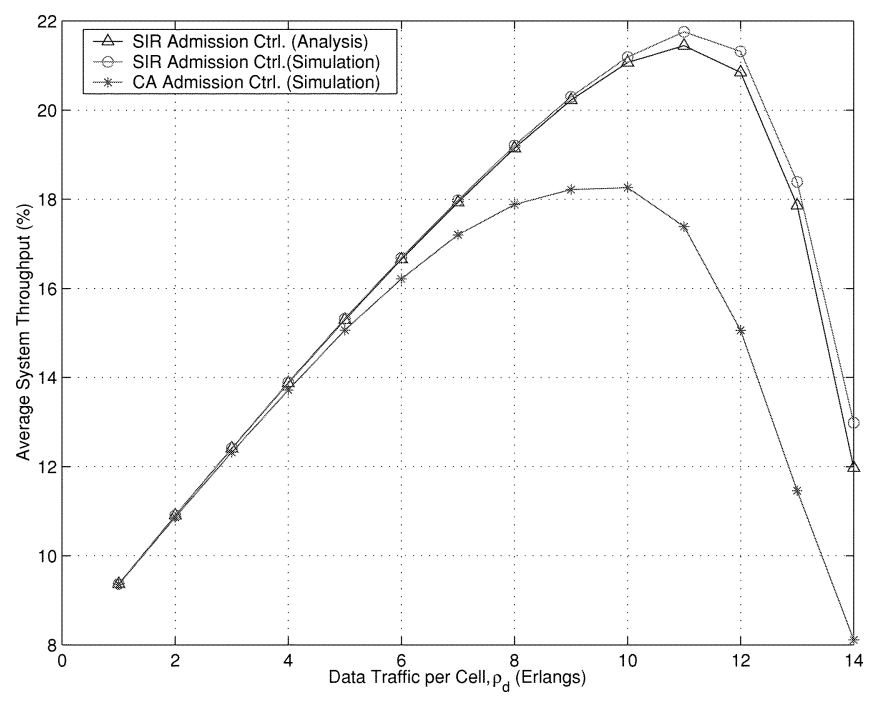

Fig. 6. Average system throughput $\eta$ versus $\rho_{d}$ for $\rho_{v}=10$ Erlangs per cell.

SIR conditions. This may allow a faster first time transmission of a data burst, but it will encounter a larger number of retransmissions due to data loss because of more interference. This results in a larger overall delay for CA-based admission control. SIR-based admission control, on the other hand, does not admit calls (i.e., buffers data bursts) if SIR conditions are not favorable. This may possibly delay the first transmission attempt more, but the transmission attempts will have a larger probability of success, because of the controlled SIR conditions. This results in a lesser overall delay compared with that of CA-based admission control. When $\rho_{v}=10$ Erlangs per cell, the mean delay performance of SIR-based admission control improves by about $40 \%$ compared with CA-based admission control, as observed in Fig. 5. This is because, at increased voice traffic loads, the CA-based admission control performs poorer because it now admits more calls (subject to code availability) than in a data-only system, which causes more retransmissions and more delay compared with SIR-based admission control.

From Figs. 4 and 5, it is also observed that at a mean delay of $50 \mathrm{~ms}$, the SIR-based admission control offers about 25\%-50\% improvement in the data Erlang capacity compared with CA-based admission control. For example, for $\rho_{v}=0$ (Fig. 4), at $\bar{D}_{d}=50 \mathrm{~ms}$, the data Erlang capacity improves from 6.5 Erlangs to 8.2 Erlangs. Similarly, for $\rho_{v}=10$ (Fig. 5), the data Erlang capacity improves from 4 Erlangs to 6 Erlangs. Fig. 6 gives the average system throughput as a function of $\rho_{d}$ for $\rho_{v}=10$ Erlangs per cell. The curve labeled "SIR Admission Ctrl. (Analysis)" in Fig. 6 is obtained by evaluating (54). We observe that because of lesser retransmission and outage probability, the SIR-based admission control utilizes the system more efficiently than the CA-based admission control.

\section{CONCLUSION}

We analyzed the performance of SIR-based admission control strategy on the uplink in cellular CDMA systems with both voice and data traffic. We presented an analytical approach to evaluate the outage probability for voice traffic, the average system throughput, and the mean delay for data traffic in a voice/data CDMA system with SIR-based admission control. The analytical results were shown to closely match with the simulation results. We showed that for a voice-only system, a capacity improvement of about $30 \%$ can be achieved with SIR-based admission control as compared with the CA-based admission control. We also showed that for a data-only system, an improvement of about $25 \%$ in both the Erlang capacity, as well as the mean delay performance can be achieved with the SIR-based admission control as compared with the CA-based admission control. Improvements of similar magnitude were shown to be achieved with SIR-based admission control in a mixed voice/data system.

\section{ACKNOWLEDGMENT}

The authors would like to thank Dr. K. N. Sivarajan, who is currently with Tejas Networks Pvt. Ltd., Bangalore, India, for his contribution in the analysis of the voice-only CDMA system.

\section{REFERENCES}

[1] A. J. Viterbi, CDMA: Principles of Spread Spectrum Communication. Reading, MA: Addison-Wesley, 1995.

[2] J. S. Evans and D. Everitt, "Effective bandwidth based admission control for multiservice CDMA cellular networks," IEEE Trans. Veh. Technol., vol. 48, pp. 36-46, Jan. 1999.

[3] C. Ho, J. A. Copeland, C. Lea, and G. L. Stuber, "On call admission control in DS/CDMA cellular networks," IEEE Trans. Veh. Technol., vol. 50, pp. 1328-1343, Nov. 2001.

[4] G. Karmani and K. N. Sivarajan, "Capacity evaluation for CDMA cellular systems," in Proc. IEEE INFOCOM 2001, Apr. 2001, pp. 601-610.

[5] N. B. Mandayam, J. M. Holtzman, and S. Barberis, "Performance and capacity of a voice/data CDMA system with variable bit rate sources," in Special Issue on Insights Into Mobile Multimedia Commun.. New York: Academic, Jan. 1997.

[6] T. Liu and J. A. Silvester, "Joint admission/congestion control for wireless CDMA systems supporting integrated services," IEEE J. Select. Areas Commun., vol. 16, pp. 845-857, Aug. 1998.

[7] W. Yue and Y. Matsumoto, "Output and delay process analysis for slotted CDMA wireless communication networks with integrated voice/data transmission," IEEE J. Select. Areas Commun., vol. 18, pp. 1245-1257, July 2000.

[8] I. Koo and K. Kim, "Erlang capacity of voice/data DS-CDMA system with prioritized services," IEICE Trans. Commun., vol. E84-B, pp. 716-726, Apr. 2001.

[9] S. Anand, A. Chockalingam, and K. N. Sivarajan, "Outage and capacity analysis of cellular CDMA with admission control," in Proc. IEEE WCNC 2002, Mar. 2002, pp. 908-912.

[10] S. Anand and A. Chockalingam, "Performance of cellular CDMA with voice/data traffic with an SIR based admission control," in Proc. IEEE GLOBECOM 2002, Nov. 2002, pp. 896-900.

[11] L. Kleinrock, Queuing Systems Volume I: Theory. New York: Wiley, 1975.

[12] L. F. Fenton, "The sum of log normal probability distributions in scatter transmission systems," IRE Trans. Commun. Systems, vol. CS-8, pp. 57-67, Mar. 1960.

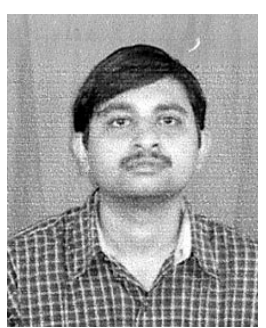

S. Anand (S'02) received the B.E. degree in electronics and communication engineering from the College of Engineering Guindy, Anna University, Chennai, India, in 1995 and the M.E. degree in electrical communication engineering from the Indian Institute of Science, Bangalore, in 1998, where he is currently working toward the Ph.D. degree.

His area of interest is radio resource management in wireless networks. 


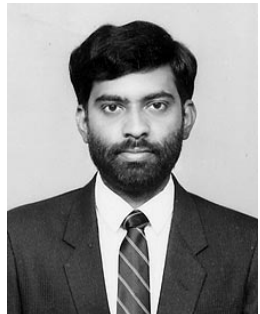

A. Chockalingam (S'92-M'93-SM'98) received the B.E. (honors) degree in electronics and communication engineering from the P.S.G. College of Technology, Coimbatore, India, in 1984, the M.Tech. degree with specialization in satellite communications from the Indian Institute of Technology, Kharagpur, India, in 1985, and the Ph.D. degree in electrical communication engineering (ECE) from the Indian Institute of Science (IISc), Bangalore, India, in 1993.

From 1986 to 1993 , he worked with the R\&D division of the Indian Telephone Industries Ltd., Bangalore. From December 1993 to May 1996, he was a Postdoctoral Fellow and an Assistant Project Scientist in the Department of Electrical and Computer Engineering, University of California, San Diego (UCSD). From May 1996 to December 1998, he served Qualcomm, Inc., San Diego, CA, as a Staff Engineer/Manager in the systems engineering group. In December 1998, he joined the faculty of the Department of Electrical Communication Engineering, Indian Institute of Science, where he is an Associate Professor, working in the area of wireless communications. He was a visiting faculty to UCSD during the summers of 1999-2002.

Dr. Chockalingam is a recipient of the Swarnajayanti Fellowship from the Department of Science and Technology, Government of India. He is an Associate Editor of the IEEE TRANSACTIONS ON VEHICULAR TECHNOLOGY. 